\title{
Electrical and Magnetic Properties of Selected Fe-Based High Entropy Alloys
}

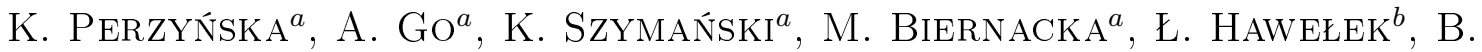

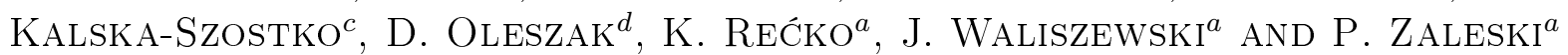 \\ ${ }^{a}$ Faculty of Physics, University of Białystok, Lipowa 41, 15-424 Białystok, Poland \\ ${ }^{b}$ Institute of Non Ferrous Metals in Gliwice, J. Sowińskiego 5, 44-100 Gliwice, Poland \\ ${ }^{c}$ Institute of Chemistry, University of Białystok, Hurtowa 1, 15-399 Białystok, Poland \\ ${ }^{d}$ Faculty of Materials Science and Engineering, Warsaw University of Technology, \\ Wołoska 141, 02-507 Warszawa, Poland
}

\begin{abstract}
The electrical and magnetic properties of $\mathrm{Fe}_{0.5} \mathrm{~A}_{0.5}$ alloys are presented, where the $\mathrm{A}$ corresponds to various compositions of the elements: $\mathrm{A}=(\mathrm{Al}, \mathrm{Si}, \mathrm{V}, \mathrm{Cr}, \mathrm{Co}, \mathrm{Ga}, \mathrm{Ni}, \mathrm{Ge})$. The system is closely related to the known family of high entropy alloys. The samples were synthesized by arc melting and/or melt spinning technique and exhibit a regular type of crystal structure. Lattice parameters are systematically smaller than the estimates based on metallic radiuses of the elements. Temperature dependences of resistivities show maxima for some compositions. This behavior is consistent with results of electronic structure calculations, where a low density of states at the Fermi level were predicted. Also the measured magnetic moments are in good agreement with results of calculations. It is thus presented that some physical properties can be designed by appropriate choice of the chemical composition within the same simple structure.
\end{abstract}

DOI: 10.12693/APhysPolA.126.999

PACS: 71.23.-k; 71.55.Ak; 72.15.-v; 72.15.Eb; 75.50.Bb; 82.80.Ej

\section{Introduction}

Structural order in the multicomponent alloys has been characterized by many orders of parameters, difficult to fully control. The limiting case of a partially ordered system is obviously a fully disordered alloy. The intention of the actual study is to investigate a system with regular structure and compositions close to some Heusler systems. Our earlier studies of a bcc $\mathrm{Fe}_{x} \mathrm{~A}_{1-x}$ system with average atom formed by $\mathrm{A}=(\mathrm{Al}, \mathrm{V}, \mathrm{Si}$ and $\mathrm{Cr})$ for a sample with $x=0.4$ [1] show the non-monotonic temperature dependence of resistivity. New family of alloys $\mathrm{Fe}_{0.5} \mathrm{~A}_{0.5}$ was prepared to investigate the influence of various compositions of the $\mathrm{A}$, as well as methods for preparing alloys with structural, electrical and magnetic properties. Our recent electronic structure calculations indicated the possibility of modifying the electronic properties by changing the A composition within the same bcc structure. In particular, a narrow pseudo-gap of density of states at the Fermi level has been theoretically predicted.

The investigated systems are closely related to the known family of high entropy alloys (HEA) [2], typically composed of more than five metallic elements of equal concentrations forming a simple, single, crystalline phase. On the other hand, our system is similar to the family of Heusler type alloys, recently intensively studied.

\section{Experimental}

The polycrystalline materials $\mathrm{Fe}_{0.5} \mathrm{~A}_{0.5}$ were prepared by repeated melting of Fe (99.95\% purity), and A $=[\mathrm{V}$,
$\mathrm{Al}, \mathrm{Si}, \mathrm{Co}, \mathrm{Ga}, \mathrm{Cr}, \mathrm{Ni}, \mathrm{Ge}$ (all $99.99 \%$ purity) in an arc furnace with argon atmosphere protection. Subsequently, parts of these ingots were melted in argon atmosphere and rapid cooled by melt spinning technique. The linear velocity of the copper wheel was $16 \mathrm{~m} / \mathrm{s}$. Compositions of the synthetized alloys are $\mathrm{Fe}_{0.5} \mathrm{Al}_{0.15} \mathrm{~V}_{0.225} \mathrm{Si}_{0.125}$ - \#1, $\mathrm{Fe}_{0.5} \mathrm{Al}_{0.125} \mathrm{~V}_{0.125} \mathrm{Si}_{0.125} \mathrm{Cr}_{0.125}-\# 2$, $\mathrm{Fe}_{0.5} \mathrm{Co}_{0.133} \mathrm{Ga}_{0.068} \mathrm{Cr}_{0.063} \mathrm{~V}_{0.061} \mathrm{Al}_{0.058} \mathrm{Ni}_{0.052} \mathrm{Ge}_{0.034} \mathrm{Si}_{0.031}$ - \#3, and methods of preparation: arc melting (am) and melt spinning (ms). Properties of samples are shown in Table I.

TABLE I

Properties of samples: lattice parameter $[\AA]-\# \mathrm{~A}$, resistivity at $30\left[10^{2} \mu \Omega \mathrm{cm}\right]-\# \mathrm{~B}$, Magnetic moment at $10 \mathrm{~K}\left[\mu_{\mathrm{B}} /\right.$ f.u. $]-\# \mathrm{C}$

\begin{tabular}{c|c|c|c|c}
\hline \hline sample & preparation & $\# \mathrm{~A}$ & $\# \mathrm{~B}$ & $\# \mathrm{C}$ \\
\hline 1-am & $\# 1, \mathrm{am}$ & $2.846(1)$ & $1.4(1)$ & $0.03(1)$ \\
2-am & $\# 2, \mathrm{am}$ & $2.845(1)$ & $4.8(1)$ & $0.18(1)$ \\
\hline 1-ms & $\# 2, \mathrm{~ms}$ & $2.847(1)$ & $1.2(1)$ & $0.23(1)$ \\
2-ms & $\# 3, \mathrm{~ms}$ & $2.874(2)$ & $3.0(3)$ & $0.78(2)$
\end{tabular}

Powder X-ray diffraction (XRD) spectra were measured at room temperature on a laboratory RigakuDenki D/MAX RAPID II-R diffractometer attached with a rotating anode $\operatorname{Ag} K_{\alpha}$ tube $(\lambda=0.5608 \AA)$, an incident beam $\left(\begin{array}{lll}0 & 0 & 2\end{array}\right)$ graphite monochromator and an image plate in the Debye-Scherrer geometry. The pixel size was $100 \mu \mathrm{m} \times 100 \mu \mathrm{m}$. Sample was placed inside glass capillaries $(0.3 \mathrm{~mm}$ in diameter $)$. Measurements were performed for both sample-filled and empty capillaries and 
the intensity for the empty capillary was then subtracted. The beam width at the sample was $0.3 \mathrm{~mm}$. The twodimensional diffraction patterns were converted into the one-dimensional intensity data using suitable software.

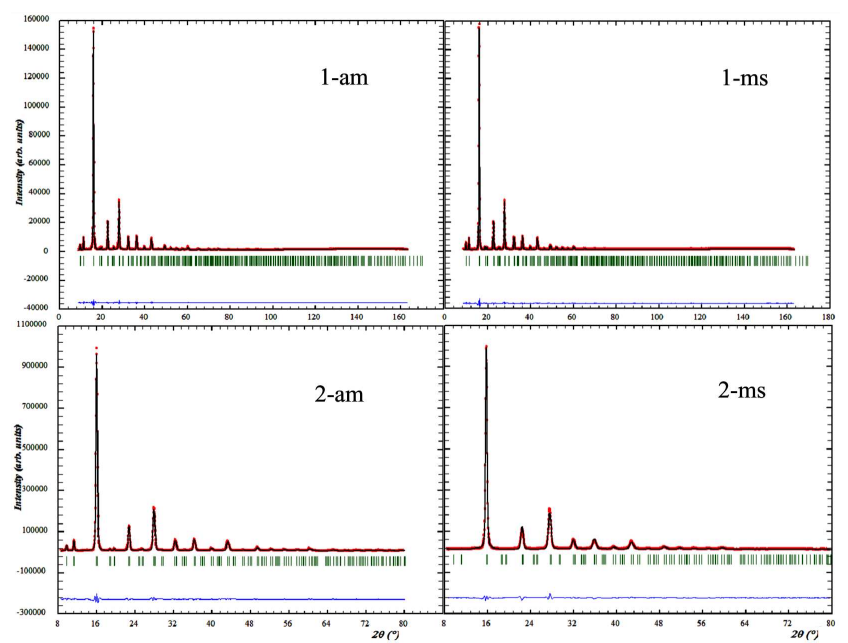

Fig. 1. Powder X-ray diffraction pattern for arc melt and melt spinning prepared samples.

In most of the samples, weak superstructure diffraction peaks are present: see, for example, Fig. 1 (1-am, 2-am), where at $2 \theta=9.8^{\circ}$ and at $2 \theta=11.3^{\circ}$, formation of $\mathrm{DO}_{3}$ type of structural order can be seen. For quantitative characteristic diffraction patterns have been refined by FullProf routine [3]. For unequivalent sublattices of the $\mathrm{DO}_{3}$-type structure, abbreviated traditionally by $(\mathrm{A}, \mathrm{C})$, $\mathrm{B}$ and $\mathrm{D}[4]$, average number of electrons were determined from the intensities of the superstructure maxima (see Table II).

TABLE II

The average number of electrons per atom in the sites $\mathrm{A}$, $\mathrm{C}, \mathrm{B}$ and $\mathrm{D}$ for $\mathrm{DO}_{3}$-type of crystalline structure.

\begin{tabular}{|c|c|c|c|c|}
\hline \multirow{2}{*}{ Sample } & \multicolumn{4}{|c|}{ Average number of electrons per atom } \\
\hline & & site $\mathrm{A}, \mathrm{C}$ & site $\mathrm{B}$ & site D \\
\hline 1-am & 21.875 & $25.64 \pm 0.24$ & $21.26 \pm 0.21$ & $14.96 \pm 0.12$ \\
\hline 2-am & 22.25 & $25.97 \pm 0.20$ & $21.91 \pm 0.25$ & $15.14 \pm 0.26$ \\
\hline $1-\mathrm{ms}$ & 22.25 & $26.07 \pm 0.29$ & $22.48 \pm 0.24$ & $14.38 \pm 0.12$ \\
\hline $2-\mathrm{ms}$ & 25.345 & $25.57 \pm 0.97$ & $25.14 \pm 1.09$ & $25.12 \pm 2.03$ \\
\hline
\end{tabular}

We had clear results that samples rich in $\mathrm{Al}, \mathrm{Si}$ and $\mathrm{V}$ (1-am, 2-am, 1-ms) show a tendency to form a $\mathrm{DO}_{3}$ type of superstructure, where three sublattices show on average, different number electrons per atom. Sample abbreviated 2-ms contains many elements with nearly equal concentration to the A component, and the formation of the superstructure is greatly suppressed.

It can be expected that the measured lattice parameter should correspond to the metallic radiuses of the components, as a consequence of empirical Vegard law.

Assume that the lattice constants $a$ of the $\mathrm{Fe}_{0.5} \mathrm{~A}_{0.5}$ alloys can been expressed as a linear combination of the

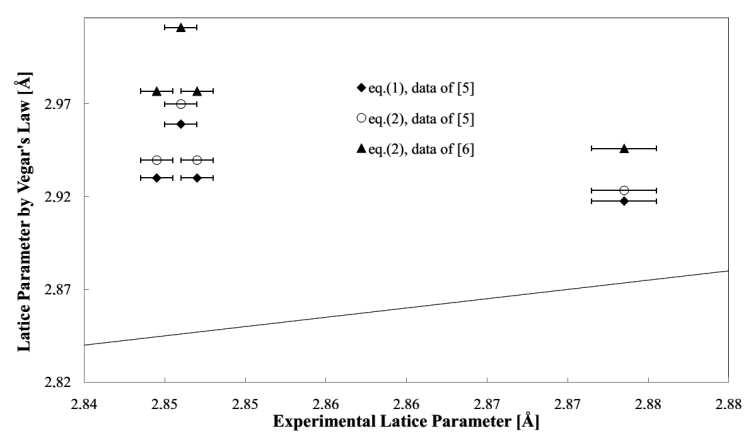

Fig. 2. Correlation between measured lattice parameters and the estimates based on metallic radiuses of the elements. Solid line corresponds to the perfect agreement of the both values.

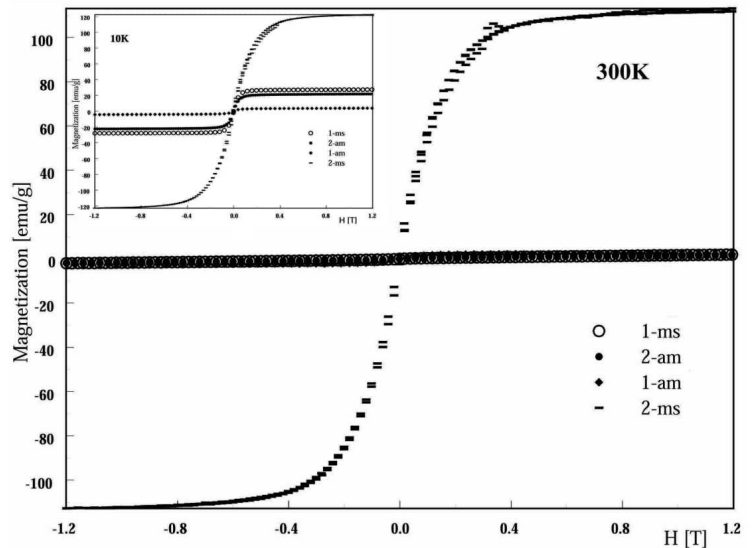

Fig. 3. Magnetization curves (M-H loops) of investigated samples at $300 \mathrm{~K}$. Inset: for comparison at $10 \mathrm{~K}$ (experimental accuracy less then size of a point).

metallic radiuses of the elements

$$
a=\frac{4}{\sqrt{3}} \sum_{i=1}^{n} n_{i} r_{i},
$$

where $n_{i}$ is a normalized molar content, while $r_{i}$ is the metallic radius of the element. Because the metallic radius in not well defined property, we present two different estimates based on the data given in $[5,6]$. It is clear that the measured values are systematically smaller by few percent than both estimates, Fig. 2. Similar results, i.e. estimates larger than measured values, are obtained when the lattice parameter is averaged by atomic volumes

$$
\bar{a}=\frac{4}{\sqrt{3}} \sqrt[3]{\sum_{i=1}^{n} n_{i} r_{i}^{3}} .
$$

We conclude that the measured parameters of our alloys are smaller by a few per cent from the estimates, irrespective of the method of estimation as well as the metallic radiuses' references (see Fig. 2). The difference ranges from 4 up to $6 \%$.

Magnetization was measured with vibrating sample magnetometer (VSM) at $10 \mathrm{~K}$ and in a magnetic field 
up to $1.2 \mathrm{~T}$ (Fig. 3). The shape of the magnetization curves indicates for soft magnetic properties. The values of spontaneous magnetization $M_{S}$ are shown in Table I.

An increase of spontaneous magnetization with an increasing variety of elements of the A, especially in Co and $\mathrm{Cr}$ has been observed. However, for samples 2-am and 1ms temperature dependences of magnetisation with magnetic field (field cooling, FC) and without magnetic field (zero field cooling, ZFC) show a ferromagnetic transition (indicated by arrows in Fig. 4). At low temperatures, where curves $\mathrm{ZFC}$ and FC do not overlap, we observe irreversibility (see Fig. 4).

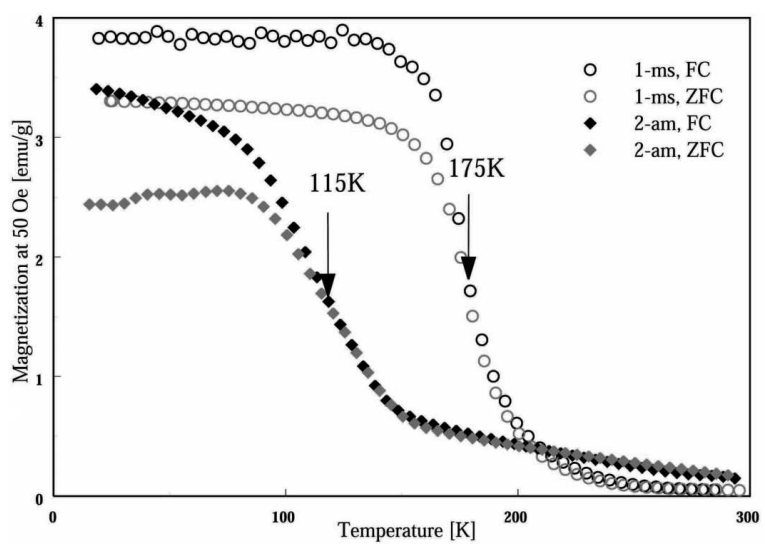

Fig. 4. Magnetization measured under FC and ZFC conditions (at $50 \mathrm{Gs}$ ) for two samples prepared by different methods (2-am, 1-ms; experimental accuracy less then size of a point). The arrows indicate the temperature of ferromagnetic transition.

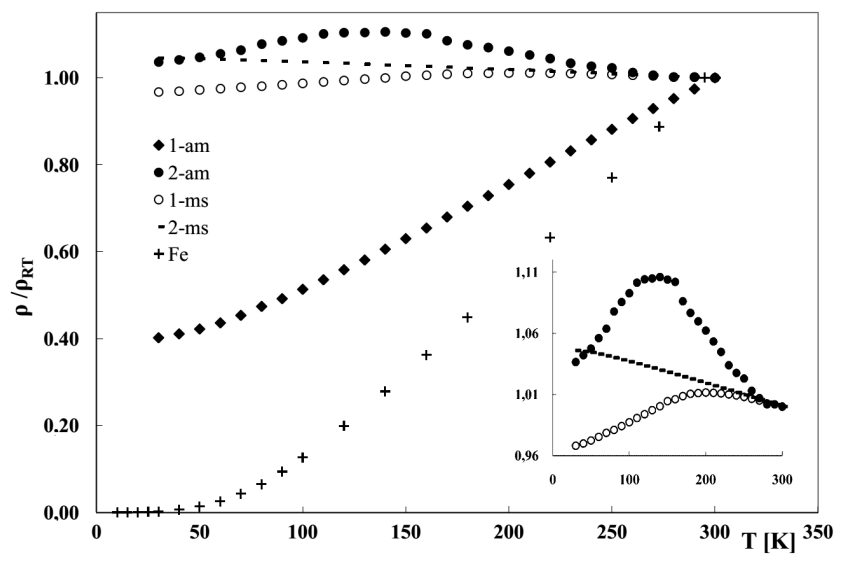

Fig. 5. Temperature dependence of resistivity in all samples. Inset: enlarged $\rho / \rho_{\mathrm{RT}}$ scale showing nontypical metallic behaviour (experimental accuracy less then size of a point). Fe data from Ref. [7] are included for comparison.

The electrical resistivity was measured by a standard dc four-terminal method over the temperature range from 24 to $300 \mathrm{~K}$. In order to check repeatability, the resistivity versus temperature was measured during the heating and cooling runs for all samples. The uncertainty of the absolute value of the resistivity was estimated to be about $5 \%$ (uncertainty in dimensions), while the reproducibility of the measurements turned out to be better than $0.5 \%$. These values have to be treated with care because we have observed in a case of sample 2-ms that three different pieces from the same ingots show differences in resistivity by factor of 2 , revealing inhomogeneities like cracks or holes.

Temperature dependence of the relative electrical resistivity $\rho(T) / \rho_{\mathrm{RT}}$ for $\mathrm{Fe}_{0.5} \mathrm{Al}_{0.15625} \mathrm{~V}_{0.21875} \mathrm{Si}_{0.125}$ am) shows an almost linear increase with temperature (Fig. 5), typical for metals. The negative slope of the resistivity can be observed for sample 2-ms, while for other samples broad maxima are observed in the $\rho(T) / \rho_{\mathrm{RT}}$. The maxima for samples prepared by the melt spinning technique are located at higher temperatures than the maximum for samples prepared by arc melting (see Fig. 5 - inset).

\section{Electronic structure calculations}

First principle spin-polarized calculations have been carried out using the full potential - linearized augmented plane waves with local orbitals $(\mathrm{FP}-\mathrm{LAPW}+\mathrm{lo})$ method implemented in Wien2k code [8]. Details of method are presented in $[1,9]$. Different concentrations of dopants: $\mathrm{Fe}_{0.5} \mathrm{Al}_{0.15625} \mathrm{~V}_{0.21875} \mathrm{Si}_{0.125}$ - \#4, $\mathrm{Fe}_{0.5} \mathrm{Al}_{0.125} \mathrm{~V}_{0.125} \mathrm{Si}_{0.125} \mathrm{Cr}_{0.125}-\#$, $\mathrm{Fe}_{0.5} \mathrm{Co}_{0.125} \mathrm{Ga}_{0.0625} \mathrm{Cr}_{0.0625} \mathrm{~V}_{0.0625} \mathrm{Al}_{0.0625} \mathrm{Ni}_{0.0625} \mathrm{Ge}_{0.03125} \mathrm{Si}_{0.03125}$ - \#6, Table III, chosen close to the experimental concentrations, were considered. Ordered and disordered structures were calculated using a supercell with 64 atoms.

TABLE III

Total magnetic moment $M_{t}\left[\mu_{\mathrm{B}} /\right.$ f.u.] calculated for compositions similar to that used in experiments at $0 \mathrm{~K}$.

\begin{tabular}{l|l}
\hline \hline Composition & $M_{t}$ \\
\hline$\# 4$ ordered & 0.033 \\
$\# 5$ ordered & 0.245 \\
$\# 5$ disordered & 0.240 \\
$\# 6$ disordered & 0.804
\end{tabular}

When $\mathrm{Fe}$ is doped with $\mathrm{Al}, \mathrm{V}$ and $\mathrm{Si}$, the alloy \#4 is almost nonmagnetic, with a total magnetic moment $0.033 \mu_{\mathrm{B}} /$ f.u. (Table III). If $\mathrm{Al}, \mathrm{V}$ and $\mathrm{Si}$ are partially replaced by $\mathrm{Cr}$, it leads to an increase of total magnetic moment to about $0.24 \mu_{\mathrm{B}} /$ f.u., almost not depending on the ordering degree of the alloy. The ordered \#5 alloy exhibits a total magnetic moment of $0.245 \mu_{\mathrm{B}} /$ f.u., while the presence of disorder slightly diminishes the total magnetic moment to the value of $0.240 \mu_{\mathrm{B}} /$ f.u. Although disorder almost does not influence on the magnetic properties of the alloy, some essential changes are visible in the density of states of \#5 near the Fermi level (Fig. 6). The last considered composition \#6 is a metallic ferromagnet with a total magnetic moment of $0.804 \mu_{\mathrm{B}} / \mathrm{f}$.u. Essentially higher than for previous compounds, the total 
magnetic moment is connected mainly with the presence of $\mathrm{Co}$ and $\mathrm{Cr}$.

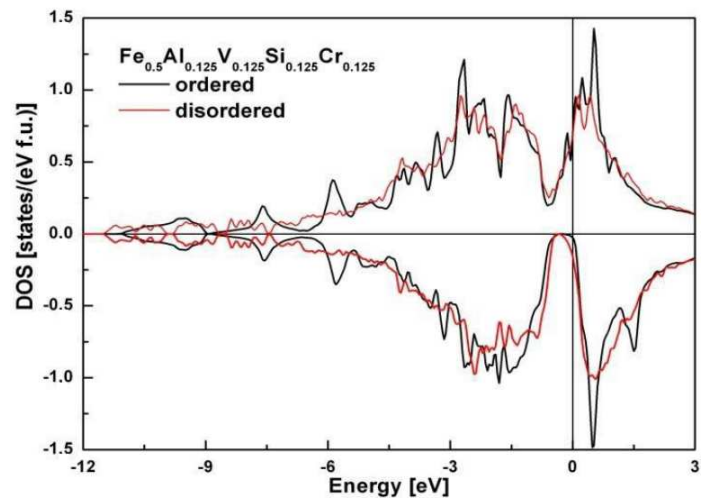

Fig. 6. Total densities of states of the ordered and disordered \#5 alloy.

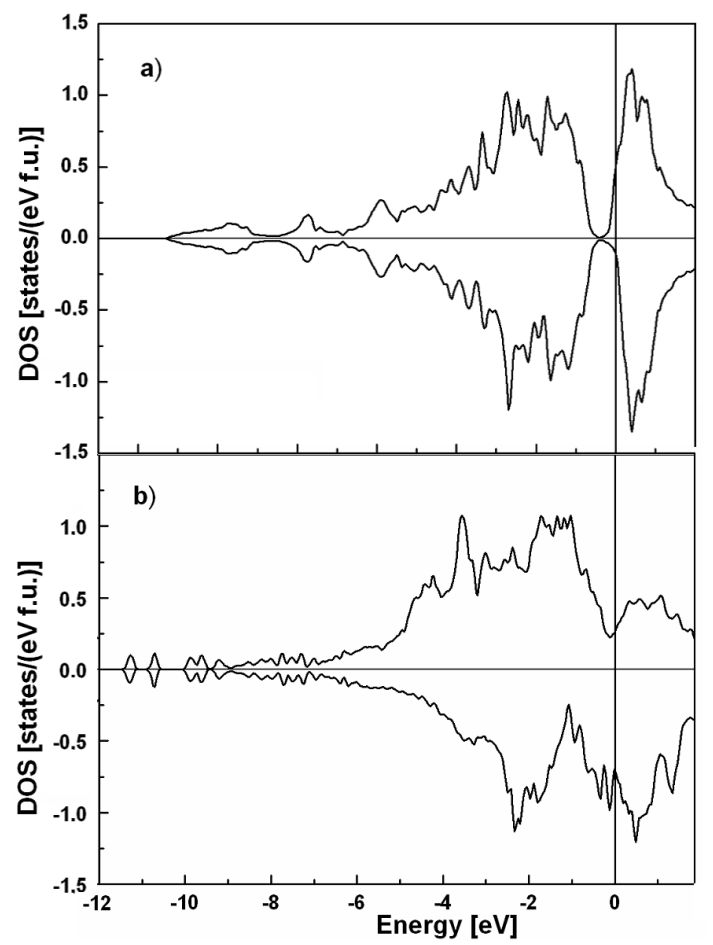

Fig. 7. Total densities of states of the alloys: a) \#4, b) $\# 6$.

The increased value of the total magnetic moment corresponds to the effect of Co DOS, which is visible particularly in Fe-Co alloys [10]. Majority Co $d$ states are almost fully occupied, whereas minority main maximum is located above the Fermi level. It leads to an increase of the spin polarization of the alloy and essentially higher total magnetic moment.

The total densities of states of \#4 exhibit pseudo-gaps below the Fermi level, which are slightly mutually shifted for the opposite spins directions (Fig. 7a). The total number of occupied majority states is almost equal to the total number of occupied minority states, thus the alloy is almost nonmagnetic. However, electronic spin polarization at the Fermi level is relatively high and equals about 0.65 . When doping atoms are partially replaced by $\mathrm{Cr}$, new majority states appear below the Fermi level (Fig. 6). If the ordered alloy is considered, the pseudogap for the minority spin channel is about 1.5 times wider than for the disordered alloy. \#6 is a metallic ferromagnet (Fig. 7b) with a complex band structure connected with the different nonequivalent surroundings of the atoms.

\section{Results and discussion}

Samples containing $50 \%$ at Fe with a regular crystal structure were synthesized and the remaining $50 \%$ of other elements serve as a kind of control parameter modifying the electronic structure. Two different methods of synthesis, arc melting and melt spinning, result in similar structural characteristics, formation of a $\mathrm{DO}_{3}$ type of superstructure enhanced by a large content of $\mathrm{Al}$, $\mathrm{Si}$ and $\mathrm{V}$ elements. Formation of the superstructure is reduced when the number of different elements in sample composition is large (sample 2-ms).

The metallic radius of an atom is an empirical rule based on the observation that the lattice parameters of the elements and compounds can be explained with reasonable precision assuming uncompressible atoms. In the case of real disordered alloys, one expects the presence of local strains and a sort of competition between individual different radiuses. Our results show that the presence of many constituents and structural disorder within the regular structure lead to apparent contraction of the lattice, by an order of a few percent. One may argue that estimates of the lattice parameter depend on the kind of averaging, and their determination is not unique. However, the estimated results presented in Fig. 2 are always larger than the measured values irrespectively of the type of averaging Eqs. (1) or (2), or the data of metallic radiuses determined by various authors. Our result remains in agreement with simulations and observations for high entropy alloys [11].

Investigated alloys are ferromagnetic in the entire temperature range. Moreover, the room temperature magnetic moments estimated from measurements are of the same order like for HEA $[12,13]$.

The temperature dependence of resistivity indicates for electron transport properties not typical for metals. This result corresponds well to the results of electronic structure calculations, where a low density of states at the Fermi level was obtained for some compositions. The correctness of the assumed theoretical model was further confirmed by agreement between magnetic moments both calculated and measured at low temperatures.

Theoretical calculations show that $\mathrm{V}$ content promotes the formation of a pseudo-gap, e.g. the low density of states at Fermi level. On the other hand, in Fe-based Heusler alloys containing V, the Fermi level is located 
at a pseudo-gap $[9,14,15]$, while the temperature dependence of resistivity is typical for metals [16-18]. The effect of the strong influence of defects on the resistivity of $\mathrm{V}[19,20]$ is known; however the role of a structural defect in explaining the observed maxima (Fig. 5 - inset) remains unclear.

The observed anomalous temperature dependence of electrical resistivity (semiconductor-like behaviour Fig. 5 - inset) is consistent with that reported by Nishino et al. [21, 22] in series of pseudobinary alloys in which Fe atoms are partly replaced by other $3 d$ transition elements. The substitution of $3 d$ elements to the left of $\mathrm{Fe}$ in the periodic table, seems to be responsible for the occurrence of the negative temperature dependence of the resistivity [21].

The appearance of the broad peak in $\rho(T) / \rho_{\mathrm{RT}}$ at 150 $180 \mathrm{~K}$ suggests that two competing mechanisms govern the electrical transport in the investigated samples. For $T<150-180 \mathrm{~K}$, we observe a positive temperature coefficient of resistivity (TRC), suggesting that $\rho(T)$ is dominated by intrinsically metallic nature. The negative slope of $\rho(T)$ for $T>120-180 \mathrm{~K}$ indicates that thermally activated behaviour is dominant due to an increase in the density of carriers across the pseudo-gap. The plots of $\ln \rho$ versus $1 / T$ ( $\rho$ - resistivity, $T$ - temperature) for the data in the temperature interval $150-300 \mathrm{~K}$ (the temperature range depends on the sample) becomes almost linear, and an energy gap $\Delta$ of about $1-10 \mathrm{meV}$ is tentatively obtained for samples: 2-am, 1-ms and 2-ms, assuming that they are semiconducting. The obtained values of the energy gap are the same as estimated by Okulov et al. [23], but significantly smaller (by one order) than ones obtained for Heusler-type alloys [19, 20, 24, 25]. The small values of $\Delta$ suggest that the densities of states of the investigated samples exhibit a pseudo-gap rather than a real energy gap. Such a result is consistent with our band structure calculations.

In a case of the sample 2-ms, which is a metallic ferromagnet with a complex band structure connected with the different nonequivalent surroundings of the atoms, the resistance maximum has disappeared, and the resistivity increases with a decrease of temperature. The resistivity reaching the value of about $300 \mu \Omega \mathrm{cm}$ at $30 \mathrm{~K}$ shows a remarkable negative temperature dependence in the whole examined temperature range.

The maximum of $\rho(T)$ for samples (e.g. 1-ms) prepared by the melt spinning technique was detected at higher temperatures than the maximum for samples (e.g. 2-am) prepared by arc melting (see Fig. 5 - inset). The temperatures of magnetic transition (marked by arrows in Fig. 4) are in good correlation with the temperature maximum on $\rho(T) / \rho_{\text {RT }}$ for these samples.

The similarity to HEA is confirmed by values of resistivity at room temperature. For all investigated samples obtained values are in good agreement with that obtained for HEA by Zhang et al. [13].

\section{Conclusions}

- Measured lattice parameters are systematically lower by a few percent than the values estimated from the metallic radiuses of the elements.

- The electronic structure calculations indicate for a possibility of tailoring electrical and magnetic properties by changing the composition within the same regular structure. In particular, a deep minimum in the density of states close to the Fermi level (a pseudo-gap) has been theoretically predicted. Furthermore, in some systems depending on composition and ordering degree, the pseudo-gap for one direction of spin was more pronounced.

- For some alloys the semiconductor-like behaviour of the temperature dependence of the electrical resistivity $\rho(T)$ has been observed. The resistivities for all investigated samples at low temperature are higher than for the pure iron and are of the same order value or higher than for HEA.

- Magnetic moments estimated from both experimental measurements and the theoretical calculations are in good agreement for all samples.

\section{Acknowledgments}

We would like to thank to Maria Tekielak for help in measurements. The work was partially supported by EU grant POW.01.03.00-20-034/09-00.

\section{References}

[1] K. Perzyńska, K. Szymański, M. Biernacka, A. Go, W. Olszewski, D. Oleszak, K. Rećko, J. Waliszewski, P. Zaleski, L. Dobrzyński, J. Phys. Soc. Jpn. 81, 064715 (2012).

[2] J.W. Yeh, S.K. Chen, S.J. Lin, J.Y. Gan, T.S. Chin, T.T. Shun, C.H. Tsau, S.Y. Chang, Adv. Eng. Mater. 6, 299 (2004).

[3] J. Rodríguez-Carvajal, Physica B Condens. Matter 192, 55 (1993).

[4] K. Szymański, M. Biernacka, L. Dobrzyński, K. Perzyńska, K. Rećko, D. Satuła, J. Waliszewski, P. Zaleski, J. Magn. Magn. Mater. 210, 150 (2000).

[5] G.E.R. Schulze, Physics of metals, Wydawnictwo Naukowe PWN, Warszawa 1982 (in Polish).

[6] C. Kittel, Introduction to Solid State Physics, 7th ed., Wiley, New York 1996, or C. Kittel, Introduction to Solid State Physics, Wydawnictwo Naukowe PWN, Warszawa 1999 (in Polish).

[7] G.K. White, S.B. Woods, Philos. Trans. R. Soc. Lond. A 251, 273 (1959).

[8] P. Blaha, K. Schwarz, G.K.H. Madsen, D. Kvasnicka, J. Luitz, WIEN2k, An Augumented Plane Wave + Local Orbitals Program for Calculating Crystal Properties, Karlheinz Schwarz, Techn. Universität Wien, Austria 2001. 
[9] A. Go, J. Solid State Chem. 219, 21 (2014).

[10] S.E. Kulkova, D.V. Valujsky, J.S. Kim, G. Lee, Y.M. Koo, Physica B 322, 236 (2002).

[11] S. Wang, Entropy 15, 5536 (2013).

[12] M.S. Lucas, L. Mauger, J.A. Munoz, Y.M. Xiao, A.O. Sheets, S.L. Semiatin, J. Horwath, Z. Turgut, J. Appl. Phys. 109, 07E307 (2011).

[13] Y. Zhang, T.T. Zuo, Y.Q. Cheng, P.K. Liaw, Nature Sci. Rep. 3, 1455 (2013).

[14] H. Okamura, J. Kawahara, T. Nanba, S. Kimura, K. Soda, U. Mizutani, Y. Nishino, M. Kato, I. Shimoyama, H. Miura, K. Fukui, K. Nakagawa, H. Nakagawa, T. Kinoshita, Phys. Rev. Lett. 8416, 3674 (2000).

[15] D.J. Singh, I.I. Mazin, Phys. Rev. B 57, 14352 (1998).

[16] J.P. Burger, M.A. Taylor, Phys. Rev. Lett. 6, 185 (1961).

[17] D.T. Hoelzer, M.K. West, S.J. Zinkle, A.F. Rowcliffe, J. Nucl. Mater. 283-287, 616 (2000).
[18] P.D. Desai, H.M. James, C.Y. Ho, J. Phys. Chem. Ref. Data 13, 1099 (1984).

[19] M. Kato, Y. Nishino, U. Mizutani, S. Asano, J. Phys. Condens. Matter 12, 1769 (2000).

[20] M. Kato, Y. Nishino, U. Mizutani, Y. Watanabe, S. Asano, J. Phys. Condens. Matter 12, 9153 (2000).

[21] Y. Nishino, M. Kato, S. Asano, K. Soda, M. Hayasaki, U. Mizutani, Phys. Rev. Lett. 79, 1909 (1997).

[22] Y. Nishino, H. Sumi, U. Mizutani, Phys. Rev. B $\mathbf{7 1}$, 094425 (2005).

[23] V.I. Okulov, V.E. Arkhipov, T.E. Govorkova, A.V. Korolyov, V.V. Marchenkov, K.A. Okulova, E.I. Shreder. H.W. Weber, Low Temp. Phys. 33, 692 (2007).

[24] Y. Nishino, S. Deguchi, U. Mizutani, Phys. Rev. B 74, 15115 (2006).

[25] T. Kanomata, T. Sasaki, T. Hoshi, T. Narita, T. Harada, H. Nishihara, T. Yoshida, R. Note, K. Koyama, H. Nojiri, T. Kanrko, M. Motokawa, J. Alloys Comp. 317-318, 390 (2001). 